\title{
Práticas educativas parentais de crianças com deficiência auditiva e de LINGUAGEM ${ }^{1}$ \\ Parental educational PRACTICES WITH THEIR CHILDREN WITH HEARING AND LANGUAge
}

IM PAIRMENTS

\author{
AlessandraTurini BOLSONI-SILVA2 \\ Olga M aria Piazentin Rolim RODRIGUES ${ }^{3}$ \\ Dagma Venturini Marques ABRA MIDES ${ }^{4}$ \\ Liliane Salgado deSOUZA ${ }^{5}$ \\ Sonia Regina LOUREIRO ${ }^{6}$
}

RESUM O: comportamentos de pais e de filhos influenciam-se mutuamente. Crianças com alterações de linguagem relacionadas ou não à perda auditiva, podem apresentar dificuldades de relacionamento com os irmãos e seus pares. Assim, a investigação das práticas educativas parentais e do repertório comportamental infantil éfundamental para a busca deintervenções ef etivas para essas crianças. O estudo tem como objetivos: (a) comparar o repertório positivo enegativo de mães e crianças com deficiência auditiva (DA) edistúrbio de linguagem (DL); (b) comparar cada uma das deficiências com grupo não clinico; (c) correlacionar comportamentos para cada uma das deficiências. Participaram desteestudo 72 mães, cujos filhos apresentavam DA $(n=27)$, DL $(n=19)$ ou compunha uma população não clínica $(n=26)$. O instrumento utilizado foi o Roteiro de Entrevista de Habilidades Sociais Educativas Parentais, que avalia a ocorrência de habilidades sociais aplicáveis às práticas educativas. Os resultados evidenciaram a associação entre práticas positivas e habilidades sociais, bem como entre práticas negativas e problemas de comportamento. O grupo de DL não apresentou mais problemas que as crianças não clínicas, sugerindo a participação de intervenções de caráter preventivo, facilitando a inclusão social. Por outro lado as crianças com DA apresentaram menos habilidades sociais, bem como suas mães, menos habilidades sociais educativas. Este estudo evidencia a importância da metodologia empregada na reabilitação de crianças com distúrbios da comunicação, sobretudo para aquelas com DA visando o beneficio de programas educativos de promoção do repertório parental positivo integrados aos objetivos da fonoterapia.

PALAVRAS-CHAVE: educação especial; família e sociedade; função educativa da família; deficiências da fala; deficiências da audição

ABSTRACT: The behavior of parents and children aremutually influential. Children with languagedisorders, whether or not they are related to hearing loss, may present difficulties in relationships with siblings and peers. Thus, the investigation of parenting practices and child behavioral repertoire is essential for finding

\footnotetext{
${ }^{1}$ Este trabal ho é parte de um trabal ho maior que culminou em Tese de Pós-Doutorado (Bolsoni-Silva, 2009), com apoio financeiro do CN Pq.

2 Pós-Doutorado, Unesp, Psicologia, Bauru - SP. bolsoni@fc.unesp.br

${ }^{3}$ Departamento dePsicologia. Faculdade de Ciências. UNESP, Bauru. olgarolim@fc.unesp.br

${ }^{4}$ Departamento de Fonoaudiologia da Faculdade de Odontologia de Bauru-USP. dagmavma@usp.br

${ }^{5}$ Departamento de Fonoaudiologia da Faculdade de Odontologia- de Bauru-USP. sal gadolili@bol.com.br

${ }^{6}$ Faculdade de Medicina de Ribeirão Preto- USP. srlourei@fmrp.usp.br
} 
effective interventions for thesechildren. This study aims to: (a) comparethe positiveand negative repertoire of mothers and children with hearing impairments ( $\mathrm{HI}$ ) and language disorder (LD); (b) compare each of the disabilities to a non-clinical population, (c) correlatebehaviors for each of the disabilities. Seventy two mothers whosechildren presented HI $(n=27)$, LD $(n=19)$, or composed a non-clinic population $(n=26)$ participated in this study. The instrument used was the Roadmap Interview Social Skills for parenting, which assesses the occurrence of social skills applicableto educational practice. The results showed positiveassociation between practices and social skills, as well as between negative practices and behavior problems. The LD group did not present more problems than non-clinical children, suggesting theinvolvement of preventiveintervention that facilitates social inclusion. On the other hand, children with $\mathrm{HI}$ presented few social skills, and their mothers, likewise, presented few social educational skills. Aiming to enhanceeducational programsthat promotepositive parental repertoire including the goals of speech-language and hearing loss therapy, this study highlights the importance of the methodology that was employed in rehabilitating children with communication disorders, particularly for those with HI.

KEYWORD S: Special Education; Family and Society; Family Educational Function; Speech-languageDisorders; Hearing Impairments.

\section{InTROdUÇÃo}

A dinâmica das relações familiares édiversificada, sofrendo influências internas e externas. O nascimento de uma criança com deficiência, tal como a auditiva ou de linguagem, exige da família uma reorganização interna impondo uma revisão de valores e de expectativas de futuro para ela e para os pais (AMIRALIAN, 1986). Considera-se que se o ambiente social permitir e prover condições, a família pode passa por um processo de superação eaceitação da criança com deficiência possibilitando a sua inclusão social e o atendimento das suas necessidades (BUSCA GLIA, 1997; SILVA; DESSEN , 2001).

Indivíduos com distúrbios da comunicação decorrentes de deficiência auditiva, de linguagem e/ ou fala, isolados ou associados a quadros clínicos de diferentes afecções neurológicas ou genéticas, podem apresentar complexas manifestações queenglobam alterações linguísticas, cognitivas, comportamentais epsi cossociais. As crianças com deficiência auditiva (DA ) podem portanto,serem consideradas como uma população de alto risco, em função da presença de indicadores, muito bem documentados, de atrasos em linguagem, em habilidades comunicativas, nas conquistas acadêmicas e no baixo ajuste sócio-emocional (CALDERON, 2000). A presença de tais dificuldades tem implicações para o desenvolvimento das crianças e para as práticas educativas adotadas pelos pais.

Na cultura brasileira, os pais utilizam enfaticamenteo comportamento verbal para interagir com os filhos, desdea mais tenra idade. Como para as crianças com deficiência auditiva tais comportamentos têm pouco efeito, os pais podem diminuir esses e de outros tipos de comportamentos comunicativos com elas, na medida em que descobrem que estas não os ouvem.

Brito eDessen (1999) afirmaram haver uma escassez de pesquisas junto às famílias de crianças com surdez, sobretudo focando as relações interpessoais. Para as referidas autoras os pais tendem a fazer pouco uso de outras funções 
comunicativas, tomando iniciativas pelos filhos, deixando poucas alternativas para a criança se desenvolver. Destacam queas mães ouvintes de crianças surdas usam mais comportamentos interativos e diretivos do que as mães surdas de crianças surdas e as mães ouvintes de crianças ouvintes.

Tais apontamentos colocam como foco a relevância das interações familiares para o desenvolvimento das crianças surdas, especial menteem função das oportuni dades que possam receber para aprender a se comunicar, sendo queo uso precoce da comunicação bimodal (oral e gestual) pode prevenir problemas e promover interações mutuamentesatisfatórias entreas mães esuas crianças surdas. N esse sentido, faz-se necessário nos estudos com crianças com deficiência auditiva, focalizar as suasfamílias.

Oliveira et al. (2004) destacaram as dificuldades comunicação enfrentadas pelas famílias de surdos que freqüentam a A npacin (A ssociação N orte Paranaense deÁudio Comunicação Infantil) , afirmando quea família podeauxiliar a criança no desenvolvimento de comportamentos de enfrentamento dos desafios. Destacam que cabe aos profissionais cuidar não apenas da criança surda, mas também de seus familiares para que possam oferecer à criança a oportunidade de se desenvolver.

Jackson (2006) relatou que o sucesso nas interações sociais depende mais da atitude dos outros do que das crianças surdas, apontando que as mesmas, por exemplo, quando são filhos de pais surdos têm menos problemas de comportamento. Todavia, o autor alerta que crianças com deficiência auditiva profunda ou severa rel ataram sentir-seisoladas, sem amigos einfel izes na escola, particularmente quando sua socialização com outras crianças com perda auditiva élimitada.

As crianças deficientes auditivas não são, necessariamente, problemáticas, mesmo com pais ouvintes, desde que algumas condições sejam consideradas, entreelas o apoio familiar, econômico esocial, segundo Fiamenghi Jr. e Messa (2007). Os referidos autores ressal tam que ter informações sobre a deficiência do filho e sobre os serviços disponíveis para o tratamento auxiliam a família no processo de desenvolvimento das habilidades sociais da criança.. Além disso, destacam como relevante a postura dos profissionais empenhados na propagação de uma idéia mais positiva e realista da deficiência. M atsukura et al . (2007) ressal taram, também, a importância do suportesocial adequado esatisfatório para as famílias com crianças com deficiência como um efeito protetivo ao bom relacionamento entre pais efilhos.

Bisol, Simioni e Sperb (2008) realizaram uma revisão de periódicos nacionais, publicados entre 1995 e 2005, enfocando as contribuições para o estudo da surdez. Os resultados indicaram que as temáticas de maior interesse são a linguagem, a língua, o desenvolvimento cognitivo e as relações familiares. A nalisando-seos tópicos relatados verifica-se que os estudos referentes às relações familiares, abordando as interações entre pais e filhos não compararam práticas 
parentais de pais de crianças com deficiências a um grupo normativo,deixando em aberto a necessidade de estudar como se desenvolvem as habilidades sociais nesse contexto.

Independenteda condição de deficiência auditiva, estudostêm relatado uma relação inversa entre o repertório de habilidades sociais eo de problemas de comportamentos (CIA; BARHAM, 2009). Considera-se que a presença de habilidades sociais promove o desenvolvimento e previne o surgimento de problemas de comportamento, favorecendo que as crianças interajam mais positivamente com pessoas de sua convivência, aumentando a chance de obterem reforçamento social, além de conseguirem resolver problemas.

Cal darella e M errell (1997) categorizaram as habilidades sociais em: 1) habilidades de relacionamentos com pares (cumprimentar, elogiar, oferecer ajuda, convidar os colegas para brincar); 2 ) habilidades de auto-controle (controlar humor, negociar, lidar com críticas); 3) habilidades acadêmicas (tirar dúvidas, seguir as orientações do professor, saber trabalhar de forma independente); 4) habilidades de ajustamento (seguir regras e instruções, usar tempo livre de forma apropriada, atender pedidos); 5) habilidades assertivas (iniciar conversação, aceitar convites, responder cumprimentos), entreoutras. Del PretteeDel Prette(2006) acrescentaram as categorias de autocontrole e expressividade emocional, civilidade, empatia, assertividade, solução de problemas interpessoais, habilidades para fazer amizades e habilidades sociais acadêmicas. Bolsoni-Silva (2008) ao propor categorias comportamentais para as habilidades sociais infantis considerou a topografia ea função, a saber: (a) Disponibilidade social e cooperação: fazer pedidos, procurar ajudar, procurar atenção, fazer perguntas, fazer elogios, cumprimentar, tomar iniciativa; (b) Expressão de sentimento e enfrentamento: expressar desejos e preferências, criticar, expressar carinho, expressar desagrado deforma habilidosa, estar de bom humor, expressar opinião, negociar, expressar os direitos e as necessidades.

Enquanto há consenso quanto à categorização das habilidades sociais, Bolsoni-Silva, Del Prettee Oishi (2003) apontaram que não há consenso quanto à denominação e classificação de problemas de comportamentos. Achenbach e Edelbrock (1979) consideraram importante avaliar a topografia de respostas e propõem a classificação dos comportamentos problemas em external izantes (por exemplo, retrai mento, depressão, ansi edadee queixas somáticas) einternal izantes (impulsividade, agressão, agitação, características desafiantes e anti-sociais). Quantos à determi nação dos problemas de comportamento os mesmos autores, a partir da revisão da literatura, listaram: variáveis históricas, características dos pais e das crianças, práticas parentais, história psiquiátrica dos pais efamiliares, relacionamento conjugal, características sócio-demográficas, relacionamento com colegas e pares.

A aceitação da multideterminação dos problemas de comportamento é clara e, segundo Patterson, Reid e Dishion (2002), as práticas parentais são 
preditivas de problemas de comportamento e, ainda que não sejam exclusivas, provavelmente são as variáveis mais importantes e, atualmente, mais documentadas. Considerando queas práticas parentais educativas são rel evantes para a promoção do desenvolvimento social das crianças, o estudo detal variável é fundamental, sobretudo quando estas apresentam alguma deficiência como a auditiva dados os poucos estudos disponíveis.

Considera-se que o desenvolvimento sócio-emocional da criança com deficiência auditiva é dependente das estratégias de comunicação desenvolvidas, principalmente na família (GA RGIULO, 2003). Para Jackson (2006) os efeitos da perda auditiva sobre o comportamento dependem das interações estabelecidas pela criança com os membros da sua família eamigos. Segundo o referido autor os pais relatam que crianças com deficiência auditiva tendem a ter mais problemas de comportamento na escola e em situação e sociais do que crianças ouvintes,possivelmente por terem dificuldade de entender e ser entendida o que resulta nos rótul os de desatenta, distraída ou imatura.

Gargiulo (2003) ressal tou que $90 \%$ das crianças com deficiência auditiva têm pais ouvintes, o que pode resultar em práticas super-protetoras, ou ainda, no distanciamento e não enfrentamento do problema.

Calderon (2000) examinou a relação entre o envolvimento de pais ouvintes com o programa escolar de seus filhos, de 42 a 90 meses de idade, com perda auditiva até 50 deci béis e as habilidades delinguagem, de leitura precoce e de adaptação sócio-emocional dos mesmos. Os resultados destetrabal ho sugerem queo envolvimento dos pais realmente contribuiu para o início da fase deleitura, sobretudo quando estavam presentes habilidades sociais comunicativas das mães.

No que se refere às crianças, sem perda auditiva, mas com al terações delinguagem, Syder (1997) indicou quea habilidadelingüística reduzida dificulta o relacionamento com os irmãos e com os pares, pois a linguagem fogeao padrão esperado. Dessa forma, a criança podetornar-se tímida, ausente, isolada, além de sofrer com os insultos de seus col egas.

Estudos têm apontado que crianças com distúrbio delinguagem (DL) podem experimentar os déficits sociais que estendem além daqueles previstos devido a seus déficits linguísticos corroborando estudos prévios queapontam que crianças com distúrbio de linguagem (DL) ecom distúrbio específico delinguagem (DEL), principalmente do tipo receptivo, desenvolvem pobremente suas habilidades sociais e correm o risco de, mais adiante, apresentarem problemas comportamentais, frágil competência social e escolar (TOMBLIN; ZHANG; BUCKWA TER, 2000; JEROME et al . 2002).

Considera-se que a natureza e gravidade das dificuldades social, emocional edecomportamento apresentadas pel as crianças com DEL são diversas e complexas, englobam ambas as dimensões, internalizante e externalizante. Crianças com este tipo deficiência exibem comportamento mais reticente do que 
os seus pares típicos, sendo que os meninos apresentam maior tendência que as meninas para o isolamento (FUJIKI et al. 1999).

Os resultados obtidos por McCabe (2005) confirmaram queas crianças com DL eDEL apresentam, significativamente, mais problemas decomportamento e pobre competência social em comparação com crianças queapresentam somente distúrbios articulatórios ou sem nenhum distúrbio de comunicação. Estes resultados foram observados tanto no ambiente doméstico quanto no escolar. As crianças com DEL demonstram particular dificuldade de orientação para as tarefas, de assertividade, baixa tolerância a frustração, mai or dependência e isolamento em sala deaula.

Com sentido semel hante, o estudo de Botting eConti-Ramsden (2008) destacou o papel complexo da linguagem nas habilidades sociais deadolescentes com histórico de deficiência delinguagem e o estudo deChiati eRoy (2008) indicou que as habilidades fonológicas e sociocognitivas precoces são preditoras dos desempenhos social e lingüístico na idade adulta, uma vez que compreendem a capacidade de responsividade social e engajamento da criança com o outro em situações do cotidiano.

Independente das dificuldades de linguagem das crianças tem-se 0 reconhecimento que os comportamentos de pais e filhos influenciam-se mutuamente e vários estudos têm apontado para tal relação. Alvarenga (2000) encontrou associação negativa entre diretividadematerna eengajamento da criança em atividades interativas. E, por outro lado, Bolsoni-Silva e Marturano $(2007,2008)$ encontraram menos problemas de comportamento em crianças cujos pais eram mais comunicativos, afetivos e estabel eciam limites com consistência.

Especificamente, quando da presença de dificuldades auditivas das crianças Motti (2000) sugeriu a necessidade de um maior investimento no atendimento aos pais a partir dos resultados de um estudo em que acompanhou 50 famílias de crianças com deficiência auditiva, menores de sete anos, periodicamente atendidas por uma equipe interdisciplinar. Verificou que eles estavam lidando mel hor com o filho eseguiam as orientações recebidas, relatando melhora na comunicação e redução dos problemas de comportamento.

Domingues, Motti e Palamin (2008) analisaram o comportamento de brincar demães ecrianças com deficiência auditiva eidentificaram quea dificuldade na comunicação impedia quea criança recebesse explicações precisas, coerentes e completas para desenvolver a percepção do que Ihe era exigido. Enfatizaram a necessidade depreparo das mães para exercer o papel deeducadora eevitar atitudes equivocadas, que não auxiliam na aprendizagem, destacando que as dificuldades maternas podem prejudicar o ensino de comportamentos social mentehabilidosos. As autoras destacaram a importância da orientação para as mães atuarem com seus filhos, por exemplo, nas formas possíveis de comunicação, para que esta seja otimizada, visando que ambos alcancem melhores níveis de desenvolvimento e adequação social. 
A análise da literatura citada atesta relação entre práticas parentais e repertório comportamental infantil, também no que se refere à presença de deficiência. Todavia, não há consenso na literatura no que se referea associação da deficiência ea maior probabilidade de problemas de comportamento. Verifica-se uma carência de pesquisas que investiguem comportamentos de pais e de filhos contingentes uns aos outros, sejam el es social mentehabilidosos ou não, assim como deestudos quecomparem práticas parentais associadas com deficiências em relação a um grupo normativo (mães de crianças sem deficiências), tais pontos justificam a proposição do presenteestudo.

Diantedo exposto os objetivos deste trabal ho são: (a) comparar práticas parentais positivas e negativas de mães de crianças com deficiência delinguagem e auditiva; (b) comparar habilidades sociais e problemas de comportamento de crianças com deficiência de linguagem e auditiva; (c) comparar cada uma das deficiências com uma população normativa, considerada não clínica por não ter qual quer deficiência e, (d) correl acionar comportamentos de mães e defil hos para cada uma das deficiências avaliadas.

\section{MÉTOdo}

\subsection{Participantes}

Participaram deste estudo 72 mães, cujos filhos apresentavam ou deficiência auditiva ( $n=27)$ (Grupo DA) ou delinguagem $(n=19)$ (Grupo DL) eas quecompunham uma amostra normativa/ não clínica $(n=26)$ (Grupo N ormativo). As crianças com deficiência auditiva (DA) foram identificadas no CEDALVI/ HRAC/ USP (Centro de Distúrbio deA udição, Linguagem eVisão, do H ospital de Reabilitação de A nomal ias Craniofaciais, da Universidade de São Paulo, Bauru), utilizavam AASI (A parel ho deA mplificação Sonora Individual) eeram filhas de pais ouvintes. Já as crianças com deficiência de linguagem (DL), na maioria dos casos caracterizada por desvio fonológico, foram rastreadas na Clínica de Fonoaudiologia da Faculdade deOdontologia de Bauru, da Universidade de São Paulo. A amostra normativa/ não clínica $(n=26)$ foi composta a partir de dois estudos: um queavaliou a efetividade de procedimento deintervenção (BOLSON ISILVA et al. 2008) e outro interessado em avaliar práticas parentais em mães separadas/ divorciadas (BOAS, 2007).

A comparação quanto as características demográficas não identificou diferenças significativas entre os grupos, apenas quanto a renda, sendo menor para o DA (Kruskal-W al lis Test eM ann-W hitney Test): (a) a idadedas crianças variou de 4a 12 anos (DA média =9,33; DL média =7,31 enão clínico média =6,08); (b) o Grupo DA é composto por 19 meninos e oito meninas; o DL é composto por 11 meninos e 8 meninas e o Grupo Normativo por 14 meninos e 12 meninas; (c) a renda é baixa, não ultrapassando, para a maioria, $R \$ 1.000,00$ (DA $=17 ; \mathrm{DL}=9 \mathrm{e}$ normativo =18); (d) a idade das mães variou de 21 a 51 anos (DA média =33; DL 
média $=39,26$ enormativo média $=31,39$ ); (e) a maioria dos participantes detodos os grupos era casada ( $D A=17, D L=17$ e normativo $=11$ ); (f) pouco mais da metade das mães $(n=39)$ relataram estarem empregadas (DA $=7$; $D L=14$ e normativo =18); ( $\mathrm{g}$ ) a escolaridade é baixa, não ultrapassando, para a maioria, o médio completo $(\mathrm{DA}=22 ; \mathrm{DL}=14$ enormativo $=20$ ).

\subsection{NSTRUMENTO}

Foi utilizado o Roteiro deEntrevista deH abilidades Sociais Educativas Parentais (RE-HSE-P, BOLSONI-SILVA, 2009), que avalia a ocorrência de seis habilidades sociais aplicáveis às práticas educativas, em escalas tipo likert com três alternativas: nunca ou quase nunca (0), al gumas vezes (1), frequentemente (2), organizadas em frequência positiva (soma de três perguntas que investigam: conversa de maneira geral, concordância parental econversa sobresexualidade) e frequência negativa que investigam:comportamento que os pais desaprovam, expressão de sentimento negativo, dificuldade em cumprir promessas) que receberam tal denominação por serem relatados contingentes ao uso de práticas positivas e negativas de educação. A princípio o instrumento investigava a freqüência das $13 \mathrm{HSE}-\mathrm{P}$, no entanto anál ises devalidade discriminativa (BOLSONISILVA, 2009) destacaram as seis HSE-P mencionadas, pois foram as que identificaram diferenças entre crianças com e sem problemas de comportamento.

A lém das perguntas fechadas, o RE-HSE-P também prevê perguntas abertas acerca de 13 habilidades sociais educativas parentais (HSE-P) que focal izam aspectos específicos das interações pais-fil hos, abrangendo não somente HSE-P, mas também práticas educativas negativas, problemas de comportamento infantis e habilidades sociais infantis. Quanto aos 13 conjuntos de informações investigase sobre os comportamentos de pais e filhos contingentes a: iniciar conversação, fazer perguntas de maneira geral, perguntar sobre sexualidade, expressar sentimentos positivos, negativos e opiniões, estabelecer limites, ter concordância conjugal quanto às práticas parentais, identificar comportamentos dos filhos que gosta e que não gosta, demonstrar carinho, cumprir promessas e identificar erros na forma de interagir com os filhos. No total, o instrumento conta com 70 itens e possui alpha de 0,846 (BOLSONI-SILVA, 2009), organizados em dois fatores: características positivas da interação (total positivo, habilidades sociais educativas, habilidades sociais infantis, variáveis de contexto e frequência positiva) e características negativas da interação (total negativo, práticas negativas, problemas decomportamento efrequência negativa). O total positivo édado pela somatória das habilidades sociais educativas parentais (HSE-P - freqüência e qualidade, habilidades sociais e variáveis de contexto) e o total negativo é a somatória de praticas parentais negativas, freqüência negativa de HSE-P e problemas de comportamento. As variáveis de contexto referem-se, por exemplo, às ocasiões em 
ocorrem a conversação (durante diferentes momentos do dia, no caminho para a escola, por exemplo), bem como os assuntos das mesmas (limites, assuntos de interesse da criança etc).

\subsection{Procedimentos de coleta de dados}

Os dados com os Grupos DA eDL foram colhidos nas próprias clínicas ecom o Grupo N ormativo os dados foram colhidos nas residências das mães e/ ou nas escolas dos seus filhos. A pós a concordância das respondentes as mesmas assinaram a um Termo de Consentimento Livre e Esclarecido. A entrevista foi conduzida conforme orientações próprias, o que implica no entrevistador fazer perguntas e registrar as respostas, para posterior categorização.

\subsection{Procedimentos de tratamento e análise de dados}

Os dad os foram computados conformeinstruções próprias (BOLSON ISILVA, 2009) e organizados nas categorias já apontadas previamente. Foram conduzidas comparações entre os grupos de deficiência auditiva e de linguagem, bem como comparações decada um desses grupos com uma população não clínica a partir do Teste U de $M$ ann-W hitney. Foram conduzidas análises de correlação (Teste Spearman) para as sub-amostras de deficiência auditiva e de linguagem. Foram considerados os resultados a partir de $5 \%$ de significância. Os itens correspondentes a freqüência negativa de HSE-P tiveram os escores invertidos para garantir a mesma direção dos dados. Os resultados estão expressos em tabelas.

\subsection{A SPECtos Éticos}

Essa amostra esta vinculada a um projeto maior denominado de "Correlacionando práticas parentais e problemas de comportamento na educação especial", cuja aprovação pelo Comitêde Ética da Faculdade deCiências da UNESP - campus Bauru, ocorreu em 11 dejaneiro de2009 na 7a- Reunião Extraordinária. A amostra da Clínica de Fonoaudiologia está vinculada ao projeto “Desempenho social decrianças com distúrbio delinguagem", aprovado pelo ComitêdeÉtica da Faculdade de Odontologia de Bauru da USP em 31 de outubro de 2007, processo $n^{\circ} 46 / 2007$.

\section{Resultados}

Essa seção apresenta os resultados obtidos nas comparações das práticas parentais, variáveis decontexto erepertório infantil dos grupos analisados de mães de crianças com deficiências auditiva (Grupo DA) e de linguagem (Grupo DL) (Tabela 1), bem como as comparações dessas amostras com uma população normativa/ não clínica (Grupo N ormativo) (Tabelas 2 e3). Final mente, encontram- 
se os resultados decorrelações (TesteSpearman ) para os dados das mães decrianças com deficiência auditiva e de linguagem (Tabelas 4 e 5). Portanto, em todas as análises foram consideradas as seguintes variáveis: (a) qualidade de habilidades sociais educativas parentais (qualidadedeHSE-P), freqüência dehabilidades sociais educativas parentais positivas (freqüência positiva deH SE-P), habilidades sociais das crianças, variáveis contextuais quesomados permitem o escore detotal positivo; (b) práticas educativas negativas, freqüência de habilidades sociais educativas parentais negativas (freqüência negativa deH SE-P) eproblemas decomportamento, que permitem obter o escore para total negativo.

Tabela 1 - Validade discriminante: médias, desvios padrão e resultados do Teste $M$ ann-W hitney (2-tailed) na comparação entreos Grupos Deficiência Auditiva (DA) x Deficiência de Linguagem (DL), no que se refere às classificações do RE-H SE-P.

\begin{tabular}{lccccc}
\hline \multirow{2}{*}{\multicolumn{1}{c}{ Categorias do RE-HSE-P }} & \multicolumn{2}{c}{ DA } & \multicolumn{2}{c}{ DL } & \multirow{2}{c}{$p$} \\
\cline { 2 - 5 } & Média & D. P. & Média & D. P. & 0,000 \\
Qualidade de HSE-P & 6,07 & 2,05 & 10,31 & 3,78 & - \\
Freqüência positiva de HSE-P & 2,81 & 1,14 & 3,18 & 1,33 & - \\
Habilidades sociais infantis & 5,70 & 2,30 & 11,00 & 4,72 & 0,000 \\
Variáveis de contexto & 5,11 & 2,49 & 10,00 & 3,11 & 0,000 \\
Total positivo & 13,63 & 3,78 & 23,77 & 7,33 & 0,000 \\
Práticas educativas negativas & 4,33 & 2,37 & 5,63 & 2,73 & - \\
Freqüiência negativa de HSE-P & 1,26 & 0,76 & 1,48 & 0,89 & - \\
\hline Problemas de comportamento infantis & 5,62 & 2,37 & 8,53 & 4,38 & 0,030 \\
Total negativo & 11,22 & 3,54 & 15,33 & 6,75 & 0,028 \\
\hline
\end{tabular}

Nota: deficiência auditiva (DA) $n=27$; deficiência de linguagem (DL) $n=19$.

A Tabela 1 indica que o tipo de deficiência implica em práticas educativas e repertórios distintos dos filhos. O Grupo DL apresenta mais características positivas deinteração (total positivo, habilidades sociais educativas, variáveis de contexto e habilidades sociais infantis) quando comparado com o Grupo DA. Por outro lado, as crianças do Grupo DL apresentam mais problemas de comportamento e total negativo que o Grupo DA. Isso também foi observado nas práticas educativas negativas que, embora mais presente nas mães do Grupo $\mathrm{DL}$, não foram estatisticamente diferentes. A Tabela 2 compara os resultados obtidos pelo Grupo DA com o Grupo Normativo. 
Tabela 2 - Médias, desvios padrão e resultados do Teste M ann-W hitney (2-tailed) na comparação entre os Grupos DA x N ormativo, no que se refereàs classificações do RE-HSE-P.

Categorias do RE-HSE-P

DA Normativo

\begin{tabular}{lccccc}
\cline { 2 - 4 } & Média & D. P. & Média & D. P. & \\
\hline Qualidade das HSE-P & 6,07 & 2,05 & 9,58 & 3,48 & 0,000 \\
Freqüência positiva de HSE-P & 1,26 & 0,76 & 1,38 & 1,13 & - \\
Habilidades sociais infantis & 5,70 & 2,30 & 10,11 & 5,00 & 0,001 \\
Variáveis de contexto & 5,11 & 2,49 & 10,46 & 6,84 & 0,001 \\
\hline Total positivo & 18,15 & 5,84 & 31,54 & 13,13 & 0,000 \\
Práticas educativas negativas & 4,33 & 2,37 & 5,88 & 3,02 & - \\
Freqüência negativa de HSE-P & 2,81 & 1,14 & 2,85 & 1,00 & - \\
Problemas de comportamento infantis & 5,62 & 2,37 & 8,23 & 4,57 & - \\
Total negativo & 12,78 & 3,29 & 16,96 & 5,85 & 0,012 \\
\hline
\end{tabular}

N ota: Grupo DA N =27 eGrupo N ormativo N $=26$.

Conforme a Tabela 2, os dados indicaram que tanto as crianças com deficiência auditiva como suas mães são menos habilidosas que o Grupo Normativo. As variáveis de contexto também indicaram interações sociais positivas ocorreram em menor número para o Grupo DA. A o contrário do esperado o total negativo foi maior para o Grupo N ormativo. Na Tabela 3 estão representados os dados comparativos entre o Grupo DL e N ormativo

Tabela 3- Médias, desvios padrão e resultados do Teste M ann-W hitney (2-tailed) na comparação entre os Grupos DL x N ormativo, no quese refere às classificações do RE-HSE-P.

Categorias do RE-HSE-P

DL Normativo

$P$

\begin{tabular}{lccccc}
\cline { 2 - 4 } & Média & D. P. & Média & D. P. & \\
\hline Qualidade das HSE-P & 10,31 & 3,78 & 9,58 & 3,48 & - \\
Freqüência positiva de HSE-P & 1,37 & 0,90 & 1,38 & 1,13 & - \\
\hline Habilidades sociais infantis & 11,00 & 4,72 & 10,11 & 5,00 & - \\
Variáveis de contexto & 10,00 & 3,11 & 10,46 & 6,84 & - \\
Total positivo & 32,68 & 10,09 & 31,54 & 13,13 & - \\
Práticas educativas negativas & 5,63 & 2,73 & 5,88 & 3,02 & - \\
Freqüência negativa de HSE-P & 3,26 & 1,24 & 2,85 & 1,01 & - \\
\hline Problemas de comportamento infantis & 8,53 & 4,38 & 8,23 & 4,57 & - \\
Total negativo & 17,42 & 6,10 & 16,96 & 5,85 & - \\
\hline
\end{tabular}

N ota: Grupo DL N = 19; Grupo N ormativo N =26. 
A Tabela 3 mostra que o Grupo DL apresentou práticas parentais e repertório infantil equival ente ao Grupo N ormativo em todos os itens aval iados. As Tabel as 4 e 5 apresentam os resultados das correlações de Spearman para os Grupos DA eDL.

Tabela 4 - Resultados da Correlação deSpearman no que se refere às classificações do RE-HSE-P para o Grupo DA. Constam os itens que tiveram pel o menos uma correlação estatisticamentesignificativa.

\begin{tabular}{|c|c|c|c|c|c|c|c|}
\hline $\begin{array}{c}\text { Categorias do RE- } \\
\text { HSE-P }\end{array}$ & 1 & 3 & 4 & 5 & 6 & 7 & 9 \\
\hline 1. Qualidade das & & $0,033^{*}$ & $0,660 * *$ & & & & \\
\hline HSE-P & & & & & & & \\
\hline $\begin{array}{l}\text { 2. Freqüência } \\
\text { positiva de HSE-P }\end{array}$ & & & & & & & \\
\hline $\begin{array}{l}\text { 3. Habilidades sociais } \\
\text { infantis }\end{array}$ & & & & $0,641^{* *}$ & & & \\
\hline $\begin{array}{l}\text { 4. Variáveis de } \\
\text { contexto }\end{array}$ & $0,660 * *$ & & & $0,795^{* *}$ & & & \\
\hline 5. Total positivo & $0,864 * *$ & $0,641 * *$ & $0,795 * *$ & & & & \\
\hline $\begin{array}{l}\text { 6. Práticas educativas } \\
\text { negativas }\end{array}$ & & & & & & $-0,430 *$ & $0,421^{*}$ \\
\hline $\begin{array}{l}\text { 7. Freqüência } \\
\text { negativa de HSE-P }\end{array}$ & & & & & $-0,430 *$ & & \\
\hline $\begin{array}{l}\text { 8. Problemas de } \\
\text { comportamento } \\
\text { infantis } \\
\text { 9. Total negativo }\end{array}$ & & & & & & & $0,795 * *$ \\
\hline
\end{tabular}

$* p<0,05 * * p<0,01$

Pela Tabela 4 nota-se que a quali dade da interação estabelecida entre mães e crianças com deficiência de linguagem foi positivamente correlacionada com as habilidades sociais das crianças ecom as variáveis decontexto (por exemplo, qualidade e diversidade da conversação). As habilidades sociais e as variáveis contextuais também foram correlacionadas com o total positivo. Por outro lado, as práticas educativas negativas, total negativo, freqüência negativa de interações e problemas de comportamento foram correlacionados entre si . 
Tabela 5 - Resultados da Correl ação deSpearman no quese refereàs classificações do RE-HSE-P para o Grupo DA. Constam os itens que tiveram pelo menos uma correlação estatisticamentesignificativa.

\begin{tabular}{|c|c|c|c|c|c|c|c|}
\hline $\begin{array}{c}\text { Categorias do RE- } \\
\text { HSE-P }\end{array}$ & 3 & 4 & 5 & 6 & 7 & 8 & 9 \\
\hline $\begin{array}{l}\text { 1. qualidade das } \\
\text { HSE-P } \\
\text { 2. frequiência } \\
\text { positiva de HSE-P }\end{array}$ & $0,512^{*}$ & $0,687^{* * *}$ & $0,834 * *$ & & $0,505^{*}$ & & \\
\hline $\begin{array}{l}\text { 3. habilidades } \\
\text { sociais infantis }\end{array}$ & & & $0,799 * *$ & & & & \\
\hline $\begin{array}{l}\text { 4. variáveis de } \\
\text { contexto }\end{array}$ & & & $0,772 * *$ & & & & \\
\hline $\begin{array}{l}\text { 5. total positivo } \\
\text { 6. práticas } \\
\text { educativas negativas }\end{array}$ & & & & & $\begin{array}{l}0,483^{*} \\
-0,462^{*}\end{array}$ & $0,530^{*}$ & $0,729 * *$ \\
\hline $\begin{array}{l}\text { 7. freqüência } \\
\text { negativa de HSE-P }\end{array}$ & & & & $-0,482 *$ & & & \\
\hline $\begin{array}{l}\text { 8. problemas de } \\
\text { comportamento } \\
\text { infantis } \\
9 \text { total negativo }\end{array}$ & & & & & & & $0,895^{* *}$ \\
\hline
\end{tabular}

$* p<0,05 * * p<0,01$

No caso da deficiência auditiva, expressas naTabela 5, a qual idade das HSE-P foram igualmente correlacionadas com habilidades sociais infantis, variáveis contextuais e total positivo. Diferentemente do esperado a qualidade dessas interações também foi correlacionada com a freqüência negativa de HSE-P. De maneira semel hante no grupo DL as práticas educativas negativas foram correlacionadas com total negativo, freqüência negativa de interações e problemas de comportamento.

De maneira geral, as correlações obtidas com o grupo com DA (Tabela 4) e com o grupo DL (Tabela 5) apontam para a relação entre habilidades sociais educativas parentais eas habilidades sociais infantis, bem como entre a existência de problema decomportamento associado a práticas educativas negativas.

\section{Discussão}

A anál ise dos resultados obtidos neste estudo evidencia a regularidade nas correlações uma vez que, independente da população há associação entre práticas positivas e habilidades sociais, bem como entre práticas negativas e problemas de comportamento (ALVARENGA, 2000; BOLSONI-SILVA; MARTURANO, 2007; BOLSONI-SILVA; MARTURANO, 2008). N estesentido, no processo de reabilitação de crianças com DA eDL os dados sugerem a rel evância 
da orientação aos pais quanto as práticas educativas positivas com o propósito de facilitar o pleno desenvolvimento da criança bem como a efetividade terapêutica das intervenções com tais grupos.

Interessante destacar que as crianças o Grupo DL não apresentaram mais problemas que as crianças do grupo normativo, sugerindo que poderiam participar de intervenções de caráter preventivo, facilitando a inclusão social. Os resultados contrariam estudos que apontaram para maiores problemas dessa população em rel ação a população sem deficiências (SYDER, 1997).

Por outro lado as crianças do grupo DA apresentaram menos habilidades sociais, bem como suas mães, que relataram, também, menos habilidades sociais educativas parentais, o que é concordante com dados prévios de outras pesquisas (CALDERON, 2000; JACKSON, 2006). No que se refere aos problemas de comportamento, o instrumento utilizado apontou os indicadores de comportamentos de desobediência e agressividade, o que recomenda para essa população grupos de suporte e acompanhamento como sugerido por Gargiulo (2003) e Motti (2000), uma vez que os grupos de suporte parental são espaços efetivos para expressar preocupações, oferecer suporte e encorajar estratégias parentais eficientes.

Estudos sobre habilidades sociais de crianças com DA, de diversas faixas etárias, relataram a eficácia de programas de intervenção na área de habilidades sociais. Entretanto, achados deWeisel, Tova eEfron (2005) apontaram queos programas educacionais regulares que integram crianças com DA com seus pares ouvintes devem estar conscientes das dificuldades sociais que ocorrem mesmo com as crianças mais novas. Por isso devem promover intervenções precoces na área deinteração social incluindo estratégias diversificadas deiniciação deinteração (englobando categorias tais como vocalização, toque neutro, toque pró-social, gestos, ato social de mostrar objetos, bater palmas, afeto positivo, expressão facial ).

Este estudo evidencia a importância da metodologia empregada no processo avaliativo de crianças com distúrbios da comunicação, sobretudo para aquelas crianças com DA visando o benefício de programas educativos de promoção do repertório parental positivo integrados aos objetivos da reabilitação fonoaudiológica.

Como limitações destacam-se a amostra reduzida, bem como a falta de outros instrumentos de aval iação, sobretudo de observação do comportamento, deforma que não se pode afirmar queo rel ato correspondeao que realmente pais e crianças fazem no seu cotidiano, o que novos estudos com metodologia observacional.

Considera-se que a investigação das práticas educativas parentais é fundamental para subsidiar a execução de intervenções mais efetivas com grupos com dificuldades específicas. N esse sentido os dados do estudo contribuem para subsidiar o processo de reabilitação eaconsel hamento dos familiares de indivíduos 
com deficiência auditiva ou de linguagem, favorecendo o atendimento integral dosmesmos.

\section{CONCLUSÕES}

A qualidade da interação estabelecida entre mães e crianças com deficiências delinguagem eauditiva foram positivamente correl acionadas com as habilidades sociais das crianças e com as variáveis de contexto, indicando que as interações estabel ecidas entre mães e filhos favorecem a aquisição e manutenção derepertório dehabilidades sociais.

O Grupo com deficiência delinguagem apresenta mais características positivas deinteração que o grupo com deficiência auditiva, inclusive de maneira equival ente ao Grupo Normativo. Já no Grupo com deficiência auditiva tanto as mães, quanto as crianças apresentaram repertório de habilidades sociais deficitário na comparação com o Grupo N ormativo. Tais achados remetem à questões importantes sobre o impacto da deficiência auditiva, entre elas a dificuldade de compreensão verbal por parte da criança, restringindo acesso direto à língua materna tendo como conseqüência o atraso de linguagem oral. Mas, convém ressaltar que esta dificul dade tendea ser compensada no processo de reabilitação com enfoquena qualidade interacional queengl oba também a linguagem não verbal por meio de pistas situacionais e da capacidade deleitura orofacial, desenvolvidas entre a díade no cotidiano. Desta forma, ainda que a comunicação mãe e filhos ocorra, o impacto emocional da DA nos pais/ mães podem interferir na qualidade interacional dificultando a responsividade materna e, até mesmo o repertorio de HS materno. No caso da deficiência auditiva, os programas de intervenção, para orientar as famílias a como interagir com seus filhos, precisam considerar esse aspecto e propor treino que engl obe o manejo de crenças e sentimentos parentais deforma a promover mel hor clima comunicacional entre pais efilhos.

Porém, não se descarta, nesse trabalho, uma possível influência sobre os resultados, da falta deoralidadeno caso da deficiência auditiva, uma vez queas mães podem secomunicar com seus filhos eesses não terem acesso ao que foi dito, prejudicando a aquisição e manutenção de repertório de habilidades sociais. No caso da deficiência au ditiva, os programas de intervenção para orientar as famílias a como interagir com seus fil hos, precisam considerar esse aspecto e propor treino adicional deforma a garantir maior comunicação entre pais efilhos.

Os resultados do estudo permitiram diferenciar as dificuldades interpessoais do grupo com deficiência auditiva em comparação ao grupo com dificuldade de linguagem confirmando, em parte, os achados da literatura apontando para maiores dificuldades interpessoais na população com alguma deficiência, indicando a necessidade de considerarem-se as especificidades dos grupos. N essesentido, a pesquisa pôde contribuir para a identificação demúltiplas variáveis tais como as habilidades sociais, os problemas de comportamento, as 
práticas parentais positivas e negativas e as variáveis contextuais em diferentes grupos de crianças, indicando que a aval iação individual izada, que leva em conta a especificidade de cada população atendida, pode favorecer a precisão do diagnóstico e nortear intervenções mais efetivas. Sugere-se estudos futuros que ampliem o número de participantes eque combinem outros métodos de coleta de dados como a observação em ambientenatural .

\section{REFERÊNCIAS}

ACHENBACH, T. M.; EDELBROCK, C. S. The child behavior profile: II. Boys aged 12-16 and girlsaged 6-11 and 12-16. Journal of Consulting and Clinical Psychology, v. 47, n. 2, p. 223233, 1979.

ALVA RENGA, P. Práticas educativas maternas eproblemas de comportamento na infância, 2000, Dissertação (M estrado em Psicologia do Desenvolvimento) - Universidade Federal do Rio Grande do Sul, Porto A legre, RS, 2000.

AMIRALIAN, M.L.T.M. Psicologia do Excepcional. São Paulo: EPU, 1986.

BISOL, C. A.; SIMIONI, J.; SPERB, T. Contribuições da psi cologia brasileira para o estudo da surdez. Psicologia: R eflexão e Crítica, Porto Alegre, v. 21, n. 3, p. 392-400, 2008.

BOAS, A.C.V.B.V. D ivórcio, habilidades sociais maternas e problemas de comportamento infantis. Dissertação (M estrado em Psi cologia do Desenvolvimento eA prendizagem), Universidade Estadual PaulistaJulio de Mesquita Filho, Bauru, 2007.

BOLSONI-SILVA, A.T. Roteiro deEntrevista deH abilidades Sociais Educativas Parentais (RE-HSE-P): Categorias e testagem preliminares. Em L.D. WEBER (Org.). Família e D esenvolvimento - Visões Interdisciplinares. Curitiba: Juruá, 2008. p. 145-158

BOLSONI-SILVA, A.T.; SA LINA, A.;VERSUTI, F.M.; ROSIN-PINOLA, A. R. A valiação de um programa de intervenção de habilidades sociais educativas parentais. Psicol ogiaCiência e Profissão, v. 28, n.1, p.18-33, 2008.

BOLSONI-SILVA , A .T.; DEL PRETTE, A .; OISHI, J. Habilidades sociais de pais eproblemas de comportamento de fil hos. R evista P sicologia A rgumento, v. 5, n. 9, p. 11-29, 2003.

BOLSONI-SILVA, A.T.; MARTURANO, E.M. A qualidade da interação positiva e da consistência parental na sua relação com problemas de comportamento de pré-escolares. Revista Interamericana de Psicologia, v. 41, n. 3, p. 349-358, 2007.

BOLSONI-SILVA, A.T.; MARTURANO, E.M. Habilidades sociais educativas parentais e problemas decomportamento: comparando pais emães de préescolares. A letheia (U LBRA ), v.27, n. 1, p. 126-138, 2008.

BOLSONI-SILVA, A. T. Estudos de confiabilidade e de validade do Roteiro de Entrevista de $\mathrm{H}$ abilidades Sociais Educativas Parentais (RE-H SE-P), 2009, 109f. (Tese de Pós-Doutorado), Faculdade de Medicina, Universidade de São Paulo, Ri beirão Preto, 2009.

BOTTING, N.; CONTI-RAMSDEN, G. The role of language, social cognition, and social skill in the functional social outcomes of young adolescents with and without a history of SLI, British Journal of D evelopmental Psychology, v. 26, p. 281-300, 2008. 
BRITO, A.M.W. ; DESSEN, M.A. Crianças surdas e suas famílias: um panorama geral. Psicologia: R eflexão e Crítica, Porto A legre, v. 12, n. 2, p. 429-445, 1999.

BUSCA GLIA, L. Os deficientes e seus pais. 3ª ed. Rio de Janeiro: Record, 1997.

CALDARELLA, P.; MERRELL, K.W. Common dimensions of social skills of children and adolescents: a taxonomy of positive behaviors. School Psychology Review, v. 26, n. 2, p. 264 278, 1997.

CALDERON , R. Parental involvement in deaf children's education programs as a predictor of child's language, early reading and social-emotional development. J. D eaf Stud D eaf E duc, v. 5, n. 2, p. 140-155, 2000.

CHIAT, S.; ROY, P. Early phonological and sociocognitive skills as predictors of later language and social communication outcomes. J ournal of C hild P sychology and P sychiatry, $v$. 49, n. 6, p. 635-645, 2008.

CIA , F.; BA RHAM , E.J. Repertório de habilidades sociais, problemas de comportamento, autoconceito e desempenho acadêmico de crianças no início da escolarização. Estudos de Psicologia, v. 26, n. 1, p. 45-55, 2009.

DEL PRETTE, Z.A.P.; DEL PRETTE, A.P sicol ogia das habilidades sociais na infância. Petrópolis: Vozes, 2006.

DOMINGUES, A.F.; MOTTI, T.F.G.; PA LAMIN, M.E.G. O brincar eas habilidades sociais na interação da criança com deficiência auditiva e mãe ouvinte. Estudos de Psicologia, Campinas, v. 25, n. 1, p. 37-44, 2008.

FIAMENGHI JR., G.A.; MESSA, A.A. Pais, filhos e deficiência: estudos sobre as relações familiares. Psicologia: Ciência eProfissão, v. 27, n. 2, p. 236-245, 2007.

FUJIKI, M. et al. Peer acceptance and friendship in children with specific language impairment. Topics in Language D isorders, v. 19, p. 34-48, 1999.

GARGIULO, R. M. Special Education in contemporary Society: an introduction to excepcional ity. Wadsworth, Thomson Learning, 2003.

JACKSON, D. Deafness and Hearing Loss. In: HEWA RD, W.L. (Org.), Exceptional children: an introduction to special education. 8. ed. New Jersey: Pearson Merrill PrenticeH all, 2006. p. 338-377

JEROME, A. et al. Self-esteem in children with language impairment. Journal of Speech, Language, and H earing R esearch, v. 45, p. 700-714, 2002.

MCCABE, P. Social and behavioral correlates of preschoolers with specific language impairment. Psychology in theSchools, v. 42, n. 4, 2005.

MATSUKURA, T.S. et al. Estresse e suporte social em mães de crianças com necessidades especiais. Revista Brasileira de Educação Especial, Marília, v. 13, n. 3, p. 415-428, 2007.

MOTTI, T.F.G.A rotina de um centro de referência em deficiência au ditiva: perspectiva depais eprofissionais. 2000. Dissertação (M estrado em Educação Especial) - UniversidadeFederal de São Carlos, São Carlos.

OLIVEIRA, R. G. et al. A experiência de famílias no convívio com a criança surda. A cta Scientiarum. H ealth Sciences, v. 26, n. 1, p. 183-191, 2004. 
PATTERSON , G.; REID, J.; DISHION , T. A ntisocial boys. Comportamento anti-social. Santo André: ESETec Editores Associados, 2002.

SILVA, N.L.P.; DESSEN, M.A. Deficiência Mental e família: implicações para o desenvolvimento da criança. Psicologia: Teoria e Pesquisa, v. 17, n. 2, p. 133-141, 2001.

SYDER, D. A n Introduction to communication disorders. São Paulo: Livraria eEditora Revinter Ltda, 1997.

TOMBLIN, J.; ZHANG, X.; BUCKWATER, P. The association of reading disability, behavioral disorders, and language impairment among second-grade children. Journal of Child Psychology and Psychiatry, v. 39, p.865-877, 2000.

WEISEL, A.; TOVA, M.; EFRON C. Iniciations of social interactions by young hearing impaired preschoolers. J. D eaf Stud D eaf E duc. Tel-A viv University, v. 10, n. 2, p.161-170, 2005. 